\title{
Factores laborales que influyen en el clima laboral del personal de enfermería del Hospital Nacional "Carlos Alberto Seguín Escobedo", Arequipa
}

\author{
Labor factors influencing the working environment of the nursing staff at the "Carlos Alberto Seguín \\ Escobedo" National Hospital
}

Milagros Lizbeth Uturunco Vera ${ }^{1}$

\section{RESUMEN}

Objetivos: Establecer los factores laborales que influyen en el clima laboral del personal de enfermería que laboran en el Hospital Nacional "Carlos Alberto Seguín Escobedo" (CASE), Arequipa. Materiales y Métodos: Es un estudio cuantitativo, correlacional y de corte transversal. La población estuvo constituida por 214 enfermeras. Los instrumentos fueron: formulario de preguntas clima laboral y Escala de Clima Organizacional (EDCO). Resultados: Los factores laborales que se dan con frecuencia en el personal de enfermería del Hospital (CASE) son la motivación en la empresa, las enfermeras afirman un $51.0 \%$ nunca les reconocen y recompensan su trabajo, $84.0 \%$ consideran que la institución no brinda estabilidad laboral, $81.0 \%$ raras veces se preocupa por su capacitación. El factor laboral motivación económica, el $45.0 \%$ consideran que el sueldo que perciben es poco adecuado, los incentivos económicos recibidos de la institución son infrecuentes un $62.0 \%$. En cuanto al factor ambiental laboral, el $49.0 \%$ consideran la relación con sus superiores es buena, $63.0 \%$ la relación con sus compañeros es buena. El factor laboral ambiente de trabajo, 55.0\% consideran el ambiente físico poco favorable y el $88.0 \%$ consideran la institución les proporcionan los recursos necesarios para llevar a cabo sus actividades sólo a veces. Conclusiones: Los factores laborales de mayor influencia en el clima laboral del personal de enfermería del Hospital (CASE), son: el reconocimiento que reciben de su institución por el trabajo bien hecho, estabilidad laboral, las relaciones con los superiores y con los compañeros de trabajo.

Palabras clave: factores laborales, clima laboral, personal de enfermería.

\begin{abstract}
Objectives: To establish the labor factors influencing the working environment of the nurses working at the "Carlos Alberto Seguin Escobedo" (CASE) National Hospital, Arequipa. Material and Methods: It is a field study, relational and cross-sectional. The population was composed of 214 nurses. The instruments were questions form work climate and organizational climate scale (EDCO). Results: The labor factors that occur frequently in the Hospital nursing staff (CASE) are the motivation in the company, the nurses claim a $51.0 \%$ never recognize and reward their work, $84.0 \%$ consider that the institution does not provide Labor stability, $81.0 \%$ rarely cares about their training. The Labor factor economic motivation, $45.0 \%$ consider that the salary they perceive is inadequate, the economic incentives received from the institution are infrequent $62.0 \%$. As for the environmental Labor factor, $49.0 \%$ consider the relationship with their superiors is good, $63.0 \%$ the relationship with their partners is Good. The work environment factor, 55.0\% Consider the physical environment unfavorable and $88.0 \%$ consider the institution provide them with the resources necessary to carry out their activities only sometimes. Conclusions: the labor factors of greatest influence in the working environment of the Hospital nurses (CASE), are: The recognition they receive from their institution for the work well done, job stability, relations with the superiors and with the Co-workers.
\end{abstract}

Keywords: labor factors, working environment, nursing staff.

${ }^{1}$ EAP de Enfermería. Universidad Privada Norbert Wiener. Lima - Perú 


\section{INTRODUCCIÓN}

Toda organización se encuentra formada por personas y las relaciones interpersonales que se dan, con el fin de desempeñar acciones que ayuden al logro de las metas. Tratar de entender el impacto que los individuos, los grupos y la estructura tienen sobre el comportamiento dentro de la organización, permite mejorar la eficacia de ésta y el alcance de sus objetivos, reflejo de ello es el clima laboral, que influyen en las actitudes y comportamiento de los empleados (1). El "clima laboral", puede ser definido como las cualidades o propiedades del ambiente laboral que son percibidas o experimentadas por los miembros de la organización y que además tienen influencia directa en los comportamientos de los empleados (2).

Las percepciones que los trabajadores y directivos se forman del ambiente lo constituye el clima laboral, el cual ejerce una influencia directa en las conductas de las personas; el clima laboral podría diferenciar a las organizaciones de éxito de las organizaciones mediocres. Mientras que un buen clima se orienta hacia los objetivos generales, un mal clima destruye el ambiente de trabajo ocasionando situaciones de conflicto y bajo rendimiento (3). La crítica situación que atraviesa actualmente el Perú, trae aparejada la difícil tarea para las instituciones de salud de revertir un Clima Laboral de tensión y frustración en uno de bienestar y satisfacción para sus empleados. Entre los factores que pueden afectar o modificar el ambiente laboral serían, las alteraciones a la estructura física de la empresa, los planes de compensaciones, beneficios y hasta la relación jefe-subordinado.

La definición del clima organizacional es "El conjunto de características permanentes que describen una organización, la distinguen de otra e influye en el comportamiento de las personas que la forman" (4).

Según la "Teoría de las Relaciones Humanas" de George Elton Mayo, concibe al trabajador no como un ente económico, sino como un ser social con sentimientos, deseos, percepciones, miedos y necesidades que lo motivan a alcanzar ciertos objetivos, donde a través de los estilos de liderazgo y las normas del grupo determinan de manera informal los niveles de producción (5). Esta teoría se relaciona con las teorías de Hildegard E. Peplau; sobre aprendizaje social, la motivación humana y el desarrollo de la personalidad donde, la Enfermera aplica los principios de Relaciones Humanas a los problemas y que se encuentra en un entorno altamente influyente Los modelos de estudio de clima laboral de las organizaciones nos plantean que existe una relación entre clima laboral y productividad de la organización. Investigaciones mostradas en el libro de Daniel Goldman "El Líder Resonante crea más", señalan: El impacto del clima laboral, sobre el rendimiento es de un $20 \%$ a un $30 \%$ (6).

Una organización con un buen clima tiene una alta probabilidad de conseguir un nivel significativo de éstos aspectos en sus miembros: en tanto, una organización cuyo clima sea deficiente no podrá esperar un alto grado de identificación. Las organizaciones que se quejan porque sus trabajadores "no tienen la camiseta puesta", normalmente tienen un muy mal clima organizacional (7).

Por lo anteriormente expuesto y considerándose que aún no se han realizado estudios en relación a los Factores laborales que influyen en el Clima Laboral en personal de enfermería del Hospital "Carlos Alberto Seguín Escobedo" - EsSalud, es que se ha interesado en realizar este trabajo de investigación y con los hallazgos encontrados se podrán generar conocimientos innovadores para mejorar el clima laboral en el Hospital.

\section{MATERIALES Y MÉTODOS}

Es un estudio de enfoque cuantitativo, de diseño no experimental, correlacional y de corte transversal. Se desarrolló en la jurisdicción del Departamento de Arequipa, específicamente en el Hospital Nacional "Carlos Alberto Seguín Escobedo" (CASE) en el distrito de Cercado, en los meses de noviembre y diciembre del 2016. La población estuvo constituida por 214 enfermeras. Los criterios de inclusión fueron: Enfermeras del sexo femenino y enfermeras de todos los servicios que acepten participar voluntariamente en la investigación. Se excluyeron: enfermeros del sexo masculino y enfermeras que no acepten participar voluntariamente en la investigación. Las técnicas de recolección de datos aplicada fue el Cuestionario, para ambas variables. Para la primera variable el instrumento fue el Formulario de Preguntas. Se entrevistó a las enfermeras en sus respectivos servicios, a la salida de su turno de trabajo.

Para el proceso de recolección de datos de la primera variable: Factores Laborales: Se utilizó el Formulario de preguntas autores: Roxana Mariela Ticona Román. Universidad Nacional de San Agustín de Arequipa - 2015. Unidad de Postgrado. Validado: Dra. Gladys Maruja Torres Condori. Ítems: 1. Motivación en la empresa. 2. Motivación 
económica. 3. Ambiente Laboral. 4. Ambiente de trabajo.

Para la segunda Variable: Clima Laboral: se utilizó el Instrumento denominado Escala de Clima Organizacional (EDCO). Los autores del instrumento son: Acero Yusset, Echeverri Lina María y Lizarazo Sandra. El instrumento utilizado fue la escala del EDCO, se refieren en una escala de frecuencias, en un continuo de cinco opciones posibles (Siempre, casi siempre, muchas veces, muy pocas veces, nunca), que desde la perspectiva estadística posee más fiabilidad que una escala de dos opciones. El instrumento está elaborado con base de 40 ítems, para cada variable se asignaron cinco ítems con actitud positiva y cinco ítems con actitud negativa. La siguiente encuesta de clima laboral - EDCO, ha sido diseñada para evaluar la situación en que se encuentra el clima laboral en las diferentes áreas y servicios en la cual laboran los trabajadores, su validez y confiabilidad esta por el cálculo del coeficiente Alpha de Cronbach, de consistencia interna, obteniéndose Alpha total "rtt" =0.9244, además de correlaciones significativas $(\mathrm{p}<0.5,01,001), \quad$ mostrando precisión y estabilidad al instrumento (8).

Para el análisis de los datos se optó por un procesamiento mixto (procesamiento electrónico y procesamiento manual), para las operaciones de la sistematización: Se realizó un plan de tabulación y un plan de codificación.

En cuanto a los aspectos éticos del estudio, se aplicaron los principios bioéticos y los criterios de protección de los participantes humanos de la investigación, en todo el proceso del estudio. A cada participante que cumplió con los criterios de inclusión, se les explico sobre el propósito del mismo y se les invitó a participar de forma voluntaria y autónoma, finalmente se les solicitó lean y firmen el consentimiento informado correspondiente.

\section{RESULTADOS Y DISCUSIÓN}

En la Tabla 1, en cuanto al factor laboral motivación en la empresa, se ha encontrado que el $51.0 \%$ afirma que "nunca" ha recibido reconocimiento y recompensa por el trabajo bien hecho y que al $49.0 \%$ solo "a veces" le reconocen el trabajo realizado. El $84.0 \%$ de las enfermeras investigadas percibe que su centro laboral no les brinda estabilidad laboral, y el $16.0 \%$ considera que si les brinda estabilidad laboral. Las enfermeras investigadas afirman que la institución en un $81.0 \%$ raras veces se preocupan brindar una adecuada capacitación, un $13.0 \%$ que nunca se preocupan y sólo el $6.0 \%$ considera que frecuentemente se preocupan por brindar una adecuada capacitación.

Tabla 1. Población de estudio según motivación en el Hospital Nacional "Carlos Alberto Seguin Escobedo", Arequipa, 2017

\begin{tabular}{|c|c|c|}
\hline \multirow[b]{2}{*}{ RECONOCIMIENTO } & \multicolumn{2}{|c|}{ TOTAL. } \\
\hline & $\mathbf{N}^{*}$ & $\%$ \\
\hline Siempre & 0 & 0.0 \\
\hline A veces & 106 & 49.0 \\
\hline Nunca & 108 & 51.0 \\
\hline \multirow[t]{2}{*}{ TOTAL } & 214 & 100.0 \\
\hline & \multicolumn{2}{|c|}{ TOTAL. } \\
\hline ESTABIUDAD LABORAL & $\mathbf{N}^{*}$ & $\%$ \\
\hline Si & 34 & 16,0 \\
\hline No & 180 & 84.0 \\
\hline \multirow[t]{2}{*}{ TOTAL } & 214 & 100.0 \\
\hline & \multicolumn{2}{|c|}{ TOTAL } \\
\hline CAPACITACIÓN & $\mathrm{N}^{*}$ & $\%$ \\
\hline Frecuentemente & 15 & 6.0 \\
\hline Rara veces & 170 & 81.0 \\
\hline Nunca & 29 & 13.0 \\
\hline TOTAL & 214 & 100.0 \\
\hline
\end{tabular}

Fuente: Elaboración propia, 2017

En la Tabla 2, en cuanto al factor laboral motivación económica, tenemos que: el $45.0 \%$ de las enfermeras investigadas considera que el sueldo que perciben actualmente es poco adecuado, el $44.0 \%$ que es inadecuado y el $11.0 \%$ que es adecuado. En cuanto a los incentivos económicos recibidos por parte de la institución, se puede evidenciar que el $62.0 \%$ señala que este se da infrecuentemente y el $38.0 \%$ que sólo se da raras veces.

Tabla 2. Población de estudio según ambiente laboral del Hospital Nacional "Carlos Alberto Seguin Escobedo", Arequipa, 2017

\begin{tabular}{lcc}
\hline \multirow{2}{*}{ SUELDO PERCIBIDO } & \multicolumn{2}{c}{ TOTAL } \\
\cline { 2 - 3 } Adecuado & $\mathbf{N}^{\circ}$ & $\%$ \\
Poco adecuado & 24 & 11.0 \\
\cline { 2 - 3 } Infrecuentemente & 98 & 45.0 \\
\hline \multicolumn{1}{c}{ TOTAL } & 92 & 44.0 \\
\hline \multirow{2}{*}{ INCENTIVOS RECIBIDOS } & $\mathbf{2 1 4}$ & $\mathbf{2}$ TOTAL \\
\hline Frecuentemente & $\mathrm{N}^{\circ}$ & $\%$ \\
Rara veces & 0 & 0.0 \\
Nunca & 81 & 38.0 \\
\hline \multicolumn{1}{c}{ TOTAL } & 133 & 62.0 \\
\hline
\end{tabular}

Fuente: Elaboración propia, 2017

En la Tabla 3, en cuanto al factor ambiente laboral, tenemos que: el $49.0 \%$ de las enfermeras investigadas consideran que la relación con sus superiores es buena, el $40.0 \%$ que es regular y el $11.0 \%$ considera que es pésima, el $63.0 \%$ de las enfermeras consideran que la relación con sus compañeros es buena, el $33.0 \%$ que es regular y el $4.0 \%$ que es pésima. 
Tabla 3. Población en estudio según ambiente laboral del Hospital Nacional "Carlos Alberto Seguin Escobedo", Arequipa, 2017.

\begin{tabular}{|c|c|c|}
\hline \multirow[b]{2}{*}{$\begin{array}{l}\text { RELACIÓN CON } \\
\text { SUPERIORES }\end{array}$} & \multicolumn{2}{|c|}{ TOTAL } \\
\hline & $\mathbf{N}^{\circ}$ & $\%$ \\
\hline Buena & 105 & 49.0 \\
\hline Regular & 85 & 40.0 \\
\hline Pésima & 24 & 11.0 \\
\hline \multirow[t]{2}{*}{ TOTAL } & 214 & 100.0 \\
\hline & \multicolumn{2}{|c|}{ TOTAL } \\
\hline $\begin{array}{l}\text { RELACIÓN CON } \\
\text { COMPAÑEROS }\end{array}$ & $\mathbf{N}^{\circ}$ & $\%$ \\
\hline Buena & 136 & 63.0 \\
\hline Regular & 68 & 33.0 \\
\hline Pésima & 10 & 4.0 \\
\hline TOTAL & 214 & 100.0 \\
\hline
\end{tabular}

Fuente: Elaboración propia, 2017

En la Tabla 4 podemos observar que la percepción del profesional de Enfermería que labora en el Hospital Nacional Carlos Alberto Seguín Escobedo - EsSalud sobre el clima laboral es neutro en un $71.0 \%$, el $17 \%$ lo percibe como negativo y un $12.0 \%$ como positivo. Lo ideal es que la gerencia propicie y facilite la evolución de los grupos de trabajo para que se conviertan en equipos de trabajo. Un grupo se convierte en equipo cuando los miembros del grupo están centrados en ayudarse entre sí para alcanzar metas de la empresa (9).

Tabla 4. Población de estudio según clima laboral del Hospital Nacional "Carlos Alberto Seguin Escobedo", Arequipa, 2017

\begin{tabular}{lcc}
\hline \multicolumn{1}{c}{ CLIMA LABORAL } & $\mathrm{N}^{\circ}$ & $\%$ \\
\hline Positivo & 26 & 12.0 \\
\cline { 2 - 3 } Neutro & 152 & 71.0 \\
\cline { 2 - 3 } Negativo & 36 & 17.0 \\
\hline \multicolumn{1}{c}{ TOTAL } & $\mathbf{2 1 4}$ & 100.0 \\
\hline
\end{tabular}

Fuente: Elaboración propia, 2017
En la Tabla 5, se puede observar que el $55.0 \%$ de la población en estudio manifiesta que el ambiente físico es poco favorable y un $29.0 \%$ que es desfavorable. La prueba estadística ji cuadrada establece que el ambiente físico no influye en el clima laboral de las enfermeras que laboran en el Hospital Nacional Carlos Alberto Seguín Escobedo.

Según HR Village: "La disposición física de un lugar de trabajo afecta directamente a la productividad de sus empleados" (10).

Se ha concluido que si una organización provee el ambiente y las condiciones adecuada para el desarrollo personal y el logro de metas y objetivos personales, las personas se comprometerán a su vez a sus metas y objetivos de la organización y se logrará la llamada integración (11).

La oportunidad de llegar a la satisfacción de las necesidades de más alto nivel es prácticamente un ideal cuando se refiere al personal trabajador que tiene que regirse por determinadas reglas que no le permiten desarrollar su talento al máximo presentando un bloqueo inminente. Esto se hace evidente cuando la empresa administra los recursos humanos sin siquiera tener presente las necesidades más básicas de los empleados, haciendo que estos abandonen todas las necesidades y dediquen a satisfacer las más básicas (12).

En el desarrollo de la investigación se pudo encontrar que la profesional de enfermería, siempre se encuentra en el medio de la organización en salud como pieza clave para el desarrollo de las actividades que en conjunto con el equipo multidisciplinario busca establecer desde su lugar un ambiente de trabajo manejable y armónico y aunque su percepción sobre ciertos temas es a veces negativa, existe la voluntad de poder cambiar y mejorar en beneficio de los pacientes.

Tabla 5. Población de estudio según ambiente físico del Hospital Nacional "Carlos Alberto Seguin Escobedo", Arequipa, 2017

\begin{tabular}{|c|c|c|c|c|c|c|c|c|}
\hline \multirow{2}{*}{$\begin{array}{c}\text { AMBIENTE } \\
\text { FISICO }\end{array}$} & \multicolumn{9}{|c|}{ CLIMA LABORAL } & \multicolumn{2}{c|}{} \\
\cline { 2 - 9 } & \multicolumn{2}{|c|}{ POSITIVO } & \multicolumn{2}{|c|}{ NEUTRO } & \multicolumn{2}{c|}{ NEGATIVO } & \multicolumn{2}{c|}{ TOTAL } \\
\cline { 2 - 9 } & $\mathbf{N}^{\circ}$ & $\%$ & $\mathbf{N}^{\circ}$ & $\%$ & $\mathbf{N}^{\circ}$ & $\%$ & $\mathbf{N}^{\circ}$ & $\%$ \\
\hline Favorable & 10 & 4.0 & 25 & 12.0 & 0 & 0.0 & 35 & 16.0 \\
\hline Poco Favorable & 16 & 8.0 & 87 & 40.0 & 15 & 7.0 & 118 & 55.0 \\
\hline Desfavorable & 0 & 0.0 & 40 & 19.0 & 21 & 10.0 & 61 & 29.0 \\
\hline TOTAL & $\mathbf{2 6}$ & $\mathbf{1 2 . 0}$ & $\mathbf{1 5 2}$ & $\mathbf{7 1 . 0}$ & $\mathbf{3 6}$ & $\mathbf{1 7}$ & $\mathbf{2 1 4}$ & $\mathbf{1 0 0}$ \\
\hline
\end{tabular}

Fuente: Elaboración propia, 2017 


\section{CONCLUSIONES}

Los factores laborales que se dan con mayor frecuencia en el personal de Enfermería del Hospital Nacional Carlos Alberto Seguín Escobedo son el factor laboral motivación en la empresa, donde las enfermeras afirman que nunca se les reconoce y recompensa su trabajo, que la institución de salud no les brinda estabilidad laboral y que raras veces se preocupa por su adecuada capacitación. En el factor laboral motivación económica, las enfermeras consideran que el sueldo que perciben es poco adecuado y que los incentivos económicos recibidos por parte de la institución son infrecuentes. En el factor ambiental laboral, las enfermeras consideran que la relación con sus superiores es buena y la relación con sus compañeros es buena. En el factor laboral ambiente de trabajo, las enfermeras consideran que el ambiente físico donde realizan sus actividades laborales es poco favorable, además consideran que la institución le proporciona los recursos necesarios para llevar a cabo sus actividades eficientemente sólo a veces.

La percepción del clima laboral que predomina es neutra en el $71 \%$ de la población en estudio, seguida por la percepción negativa en el $17 \%$. Existen algunos indicios de conformismo, apatía, resignación y aceptación de la autoridad, sin importar la integración entre las actividades de la organización y las necesidades personales.

Los factores de mayor influencia en el clima laboral del personal de enfermería del Hospital Nacional Carlos Alberto Seguín Escobedo, son: el reconocimiento que reciben de su institución por el trabajo bien hecho, estabilidad laboral, las relaciones con los superiores y con los compañeros de trabajo.

\section{AUTOR DE CORRESPONDENCIA}

Milagros Lizbeth Uturunco Vera

EAP de Enfermería

Universidad Privada Norbert Wiener

Dirección:

Teléfono:

\section{REFERENCIAS BIBLIOGRÁFICAS}

1. MAGALHAES M, MERCADO F. Investigación cualitativa en los servicios de salud. Argentina. Editorial lugar. 2007.

2. MALAGÓN L. Administración Hospitalaria. Colombia. Editorial Médica Panamericana. Tercera edición. 2006.
3. PAREDES J. Diseño de investigación científica. Perú. Editorial Latinoamericana. 2000.

4. BRUNET L. El clima de trabajo en las organizaciones definiciones, diagnósticos y consecuencias. México. Editorial Trillas. 2009

5. PÉREZ F. Guía práctica de desarrollo organizacional. México. Editorial Trillas. 2008.

6. PHEGAN B. Desarrollo de la cultura de su empresa. México. Editorial Panorama. 2006.

7. GRUNER L. Poder y desarrollo organizacional. México. Editorial Addison Wesley iberoamericana. 2009 pp. 45-47

8. HIDALGO J. El clima organizacional afecta en el desempeño laboral de la Empresa Importador Ferretero Trujillo CIA. Ltda. en la matriz en la ciudad de Quito" [Acceso el 13 de mayo de 2016] [Internet].

9. Artículo Virtual. Clima Organizacional Inavi Falcón. 3/6/2014. [Acceso el 25 Junio de 2016] [Internet].

10. IVANCEVICH J, KONOPARK R. Comportamiento organizacional. México. Ediciones Mc Graw-Hill interamericana. Sétima edición. 2006, [Acceso el 25 de mayo de 2016] [Internet].

11. GARCÍA J, JIMÉNEZ F, ARNAUD R. Introducción a la metodología de la investigación en Ciencias de la Salud. México. Ediciones Mc Graw-Hill interamericana. 2011.

12. LÓPEZ C, NAVARRO J. Análisis del clima organizacional aplicado al hospital de Victoria Valdivia. Chile. Universidad Austral de Chile, Facultad de ciencias económicas y administrativas. 2008. [Acceso el 21 de mayo de 2016] [Internet]. 